\title{
Size and mechanics effects in surface-induced melting of nanoparticles
}

\author{
Valery I Levitas ${ }^{1} \&$ Kamran Samani²
}

Various melting-related phenomena (like surface melting, size dependence of melting temperature, melting of few nm-size particles and overheating at a very fast heating rate) are of great fundamental and applied interest, although the corresponding theory is still lacking. Here we develop an advanced phase-field theory of melting coupled to mechanics, which resolves numerous existing contradictions and allowed us to reveal exciting features of melting problems. The necessity of introducing an unexpected concept, namely, coherent solid-melt interface with uniaxial transformation strain, is demonstrated. A crossover in temperature dependence of interface energy for radii below $20 \mathrm{~nm}$ is found. Surface-induced premelting and barrierless melt nucleation for nanoparticles down to $1 \mathrm{~nm}$ radius is studied, and the importance of advanced mechanics is demonstrated. Our model describes well experimental data on the width of the molten layer versus temperature for the Al plane surface and on melting temperature versus particle radius.

\footnotetext{
1 Departments of Aerospace Engineering, Mechanical Engineering and Material Science and Engineering, lowa State University, Ames, lowa 50011, USA. 2 Department of Mechanical Engineering, lowa State University, Ames, lowa 50011, USA. Correspondence and requests for materials should be addressed to V.I.L. (vlevitas@iastate.edu).
} 
$\mathrm{N}$ umerous melting-related phenomena represent fundamental material problems and are currently under intense experimental and theoretical study. They include surface premelting and melting below the thermodynamic melting temperature $\theta_{\mathrm{e}}$, caused by reduction in surface energy and leading to appearance of a molten, nanometer-thick layer ${ }^{1,2}$; reduction in melting temperature $\theta_{\mathrm{m}}$ with reduction of the particle radius $R$ down to nanoscale ${ }^{3,4}$; melting of particles with radii comparable to and smaller than the equilibrium solid-liquid interface width $\delta_{\mathrm{e}}$, which is a few $\mathrm{nm}^{3,5}$; and overheating above $\theta_{\mathrm{e}}$ during very fast heating ${ }^{6,7}$. All of these phenomena allow one to determine the properties of solid and liquid deeply in the region of their metastability and even complete instability (that is, above the solid instability temperature $\theta_{\mathrm{i}}$ or below the melt instability temperature $\theta_{c}$, see Supplementary Fig. S1), and to study intermediate states, various scale effects and non-equilibrium thermodynamic and kinetic properties. These studies also have important applied aspects, for example, for combustion of $\mathrm{Al}$ nanoparticles ${ }^{8}$, which are the most known representatives of nanoenergetic materials. However, a consistent theoretical framework for the description of the above phenomena is lacking. The sharp-interface approach ${ }^{9}$ (SIA) is not justified for the above problems. Although there are important molecular dynamics (MD) studies $^{5,6,10}$, we focus on the continuum phase-field approach (PFA), which allows consideration of larger spatial and time scales and operates explicitly with thermodynamic and kinetic parameters determined at the macroscale (see Supplementary Discussion). When mechanics is taken into account, a basic problem of the description of finite-width, solid-liquid interface appears. Traditionally, solids and liquids are described in completely different continuum mechanical frameworks (for example, solids are described in undeformed states, whereas liquids are described in a deformed state), which sophisticates the description of intermediate state. Some works consider solid as a very viscous liquid ${ }^{13}$, that is, neglect elastic shear modulus $\mu$. Such a liquid-liquid interface is incoherent and does not generate internal elastic stresses, similar to sharp interface (Fig. 1). Alternatively ${ }^{14}$, solid-melt interface is considered as a coherent interface (Fig. 1), in which shear modulus varies from that for solid to zero, and this results in generation of internal elastic stresses at the interface. When particle size is comparable to or smaller than the interface width, a coherent interface is the only reasonable choice. Although this model is supposed to be more precise, the surface tension in it is not consistent with sharp-interface limit, even if $\mu=0$. Also, even for liquid-liquid interface, expression for surface tension in PFA differs by a hydrostatic pressure from that in SIA ${ }^{11-13}$. This is a fundamental thermodynamic inconsistency, which is especially important for nanoscale interface radii.

Surface premelting and melting were studied using PFA $^{15-17}$ without involving mechanics. However, the employed equation for surface energy, $\gamma(\eta)=a+b \eta^{2}$, did not allow a homogeneous solution for solid $(\eta=1)$ (Fig. 2), exhibiting a surface disordered structure even below melt instability temperature $\theta_{c}$, when the energy minimum corresponding to melt does not exist. Such an inconsistency also exhibits itself in unphysical regions in the phase diagram ${ }^{15}$.

The goal of this paper is to develop a unified PFA coupled to mechanics and reveal the non-trivial features of the above meltrelated phenomena. Thus, a model with coherent solid-melt interface is developed (see Fig. 1), in which shear modulus $\mu$ varies from that for solid to zero, and this results in generation of internal elastic stresses at the interface. Thermodynamic potential in this model results in surface-tension stresses consistent with the SIA, in contrast to known models with incoherent interface (for which $\mu=0)^{11-13}$ and coherent interface ${ }^{14}$. Also, the necessity of introducing a non-spherical transformation strain, which, in addition to volumetric expansion during melting, describes change in shape, is demonstrated. Our model resolves fundamental thermodynamic inconsistency and includes more advanced mechanics, which is especially important
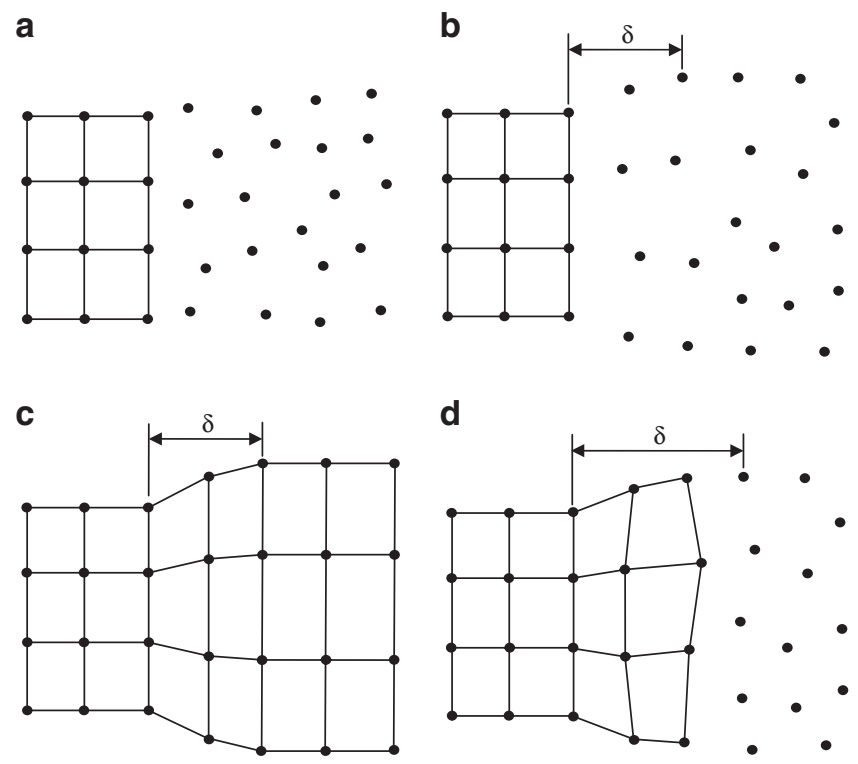

Figure 1 | Different types of interfaces between solid and molten phases. (a) Traditional sharp incoherent solid-melt interface, which does not generate internal elastic stresses. (b) Incoherent, finite-width, solid-melt interface with zero shear modulus $\mu=0$ that does not generate internal elastic stresses. (c) Coherent finite-width interface between two solid phases. Continuity of crystal lattice across the interface along with a change in the size of one of the lattices during transformation (due to transformation strain tensor $\varepsilon_{t}$ ) generates internal elastic stresses. (d) Coherent, finite-width, solid-melt interface with non-zero shear modulus $\mu \neq 0$ that generates internal elastic stresses.

for nanoparticles. Also, a model for surface energy that varies during melting is developed, which resolves the aforementioned nonphysical effects in existing models ${ }^{15-17}$ and adds mechanics to them. It is demonstrated that our model describes well experimental data on the width of the molten layer versus temperature for the Al plane surface, and then it is applied to surface-induced premelting and barrierless melt nucleation for nanoparticles. It also describes well experimental data on $\theta_{\mathrm{m}}$ versus $R$, even better than MD simulations ${ }^{5}$. Then, non-equilibrium and small-scale regimes were treated, that is, interface profile, width, energy and velocity have been determined and analysed versus $R$, interface position $r_{i}$, and large overheating and heating rates $\hbar$. Thus, the derived analytical expression for interface velocity $v$ describes well simulation results well outside an expected range, namely, even for $\theta>\theta_{i}, \hbar$ up to $10^{13} \mathrm{~K} \mathrm{~s}^{-1}$, and $r_{i} \geq 2 \delta_{\mathrm{e}}$. It also describes well the effect of large compressive and tensile pressure. Homogeneous melt nucleation competes for $\hbar=10^{13} \mathrm{~K} \mathrm{~s}^{-1}$ with interface propagation. For $R=1$ and $3 \mathrm{~nm}$, premelting occurs in the entire particle rather than at the surface only. Allowing for mechanics increases the melting temperature by $70 \mathrm{~K}$ for $R=1 \mathrm{~nm}$ particle due to pressure induced by surface tension. For $R \leq 1.5 \mathrm{~nm}, \theta_{\mathrm{m}}<\theta_{\mathrm{c}}$, that is, the particle melts more than $200 \mathrm{~K}$ below $\theta_{\mathrm{e}}$, while there is no local energy minimum corresponding to melt. While for a liquidliquid interface our calculations reproduce the Laplace relationship for jump in radial stresses $\Delta \sigma_{\mathrm{r}}$, for coherent solid-melt interface, $\Delta \sigma_{\mathrm{r}}$ does not follow the Laplace relationship and even may possess the opposite sign, causing tension in the solid core instead of compression. Interface energy varies in a non-trivial way for $r_{i} \leq 4 \delta_{\mathrm{e}} \simeq 12 \mathrm{~nm}$ with decreasing $r_{i}$, increasing for $\theta>\theta_{\mathrm{e}}$ and decreasing for $\theta<\theta_{\mathrm{e}}$; this is opposite to the behaviour for $r_{i}>12 \mathrm{~nm}$.

\section{Results}

Theory. We designate contractions of tensors $\boldsymbol{A}=\left\{A_{i j}\right\}$ and $\boldsymbol{B}=\left\{B_{j i}\right\}$ over one and two indices as $\boldsymbol{A} \cdot \boldsymbol{B}=\left\{A_{i j} B_{j k}\right\}$ and $\boldsymbol{A}: \boldsymbol{B}=A_{i j} B_{j i}$, 


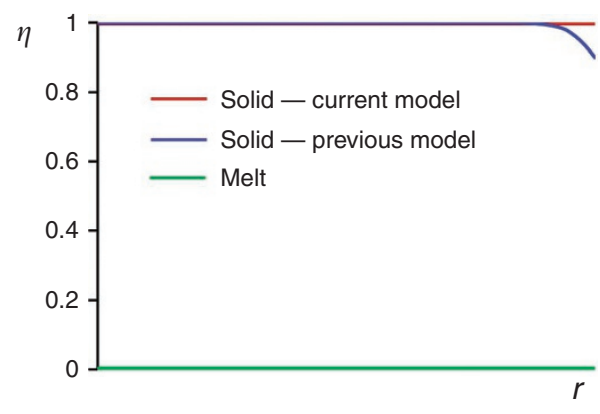

Figure 2 | Stationary solutions for Ginzburg-Landau equation with different models for surface energy. Previous models ${ }^{15-17}$ (blue line) did not allow a homogeneous solution for the solid $(\eta=1)$, exhibiting a surface disordered structure at any temperature. Our model equation (8) (red line) is developed using the condition that it allows a homogeneous solution for the solid. The green line represents melt $(\eta=0)$.

respectively. The subscript ${ }^{*}$ means symmetrization; the subscripts or superscripts $e, \theta$ and $t$ are for elastic, thermal and transformational contributions to strain and energy; $I$ is the unit tensor; $\Delta A=A_{\mathrm{s}}-A_{\mathrm{m}}$ for any property $A$, with subscripts s and $m$ denoting solid and melt, respectively; $\nabla$ and $\nabla$ are the gradient operators in the undeformed and deformed states; and $\otimes$ designates a dyadic product.

To develop a unified approach to solid and liquid, we will consider liquid as the limit case of isotropic viscoelastic solid with $\mu=0$. For simplicity, viscosity is neglected, isothermal processes are considered and shear strain is small. However, even if volumetric strain $\varepsilon_{0}$ is small, in order to reproduce surface tension one has to use fully large-strain formulation (see below). We will modify our recently developed theory of martensitic transformations in solids ${ }^{18,19}$ to describe melting. The Helmholtz free energy per unit undeformed volume of solid $\psi=\psi(\varepsilon, \eta, \nabla \eta, \theta)$, in which $\boldsymbol{\varepsilon}$ is the strain tensor and $\eta$ is the order parameter that varies from 1 in solid to 0 in melt. Using an irreversible thermodynamic procedure based on the application of the first and second laws of thermodynamics to the system with energy depending on the gradient of the order parameter (see ref. 12 and references in it) and assuming linear relation between thermodynamic force and flux, one obtains expression for the stress tensor $\boldsymbol{\sigma}$ and the Ginzburg-Landau (GL) equation

$$
\begin{aligned}
\sigma & =\frac{\partial \psi}{\partial \boldsymbol{\varepsilon}}-J^{-1}\left(\nabla \eta \otimes \frac{\partial \psi}{\partial \nabla \eta}\right)_{*}, \\
\frac{1}{\chi} \frac{\partial \eta}{\partial t} & =-\left.J^{-1} \frac{\partial \psi}{\partial \eta}\right|_{\varepsilon}+\nabla \cdot\left(J^{-1} \frac{\partial \psi}{\partial \nabla \eta}\right), \quad J=\frac{\rho_{0}}{\rho}=1+\varepsilon_{0},
\end{aligned}
$$

in which $\rho_{0}$ and $\rho$ are the mass densities in the non-deformed and deformed states, $\chi$ is the kinetic coefficient, and $\varepsilon=$ const while evaluating $\partial \psi / \partial \eta$. The kinematics relationship between displacement $\boldsymbol{u}$ and strain $\varepsilon=1 / 3 \varepsilon_{0} \boldsymbol{I}+\boldsymbol{e}$, decomposition of $\varepsilon$ and the equilibrium equation are given by

$$
\varepsilon=(\stackrel{\circ}{\nabla} u)_{\star}, \quad \varepsilon=\varepsilon_{\mathrm{e}}+\varepsilon_{\mathrm{t}}+\varepsilon_{\theta}, \quad \nabla \cdot \sigma=0,
$$

in which $\boldsymbol{e}$ is the deviatoric strain. Energies and strains are defined as follows:

$$
\begin{gathered}
\psi=\psi^{\mathrm{e}}\left(\varepsilon_{0}, \boldsymbol{e}, \eta, \theta\right)+J \breve{\psi}^{\theta}+\psi^{\theta}+J \psi^{\nabla} ; \\
\psi^{\mathrm{e}}=0.5\left(K_{\mathrm{m}}+\Delta K \phi(\eta)\right) \varepsilon_{0 \mathrm{e}}^{2}+0.5 \mu \phi(\eta) \boldsymbol{e}_{\mathrm{e}}: \boldsymbol{e}_{\mathrm{e}} ; \\
\psi^{\theta}=H\left(\theta / \theta_{\mathrm{e}}-1\right) \phi(\eta) ; \breve{\psi}^{\theta}=A \eta^{2}(1-\eta)^{2} ; \\
\psi^{\nabla}=0.5 \beta|\nabla \eta|^{2} ; A:=3 H\left(1-\theta_{\mathrm{c}} / \theta_{\mathrm{e}}\right) ;
\end{gathered}
$$

$$
\left.\varepsilon_{\mathrm{t}}=\bar{\varepsilon}_{\mathrm{t}}(1-\phi(\eta)) ; \quad \varepsilon_{\theta}=\left(\alpha_{\mathrm{m}}+\Delta \alpha \phi(\eta)\right)\left(\theta-\theta_{\mathrm{e}}\right)\right) \boldsymbol{I} ; \quad \phi(\eta)=\eta^{2}(3-2 \eta)
$$

Here $K$ is the bulk modulus, $\beta$ and $\alpha$ are the gradient energy and linear thermal expansion coefficients, $H$ is the heat of fusion, $\breve{\psi}^{\theta}$ is the double-well energy, and $\overline{\boldsymbol{\varepsilon}}_{\mathrm{t}}$ is the transformation strain that transforms the elemental volume of solid to melt under $\boldsymbol{\sigma}=0$. While for martensitic phase transformations $\overline{\boldsymbol{\varepsilon}}_{\mathrm{t}}$ is a tensor connecting two crystal lattices, for melting it is always pure volumetric strain, $\overline{\boldsymbol{\varepsilon}}_{\mathrm{t}}=1 / 3 \varepsilon_{0 \mathrm{t}} \boldsymbol{I}^{9,11-14}$. We will show that the usual assumption causes very high internal stresses and elastic energy within the interface, which suppresses melt nucleation and contradicts with the experiment. Development of a complete theory for $\overline{\boldsymbol{\varepsilon}}_{\mathrm{t}}$ is not a goal of this paper, but we consider an alternative expression for uniaxial $\bar{\varepsilon}_{\mathrm{t}}=\varepsilon_{0 \mathrm{t}} \boldsymbol{k} \otimes \boldsymbol{k}$, in which $\boldsymbol{k}=\nabla \eta /|\nabla \eta|$ is the unit normal to the interface. In this case, the component of transformation strain along the interface is absent, which minimizes internal stresses and their energy. Then equation (1) looks like

$$
\begin{aligned}
& \boldsymbol{\sigma}=\boldsymbol{\sigma}_{\mathrm{e}}+\boldsymbol{\sigma}_{\mathrm{st}} ; \quad \boldsymbol{\sigma}_{\mathrm{st}}=\left(\psi^{\nabla}+\breve{\psi}^{\theta}\right) \boldsymbol{I}-\beta \nabla \eta \otimes \nabla \eta \\
& \boldsymbol{\sigma}_{\mathrm{e}}=\left(K_{\mathrm{m}}+\Delta K \phi(\eta)\right) \varepsilon_{0 \mathrm{e}}+\mu \phi(\eta) \boldsymbol{e}_{\mathrm{e}} ; \\
& \frac{1}{\chi} \frac{\partial \eta}{\partial t}=\beta \nabla^{2} \eta-6 J^{-1}\left[H\left(\frac{\theta}{\theta_{\mathrm{e}}}-1\right)+\sigma^{\mathrm{e}} \varepsilon_{0 \mathrm{t}}-3 p_{\mathrm{e}} \Delta \alpha\left(\theta-\theta_{\mathrm{e}}\right)\right. \\
& \left.+\frac{1}{2} \Delta K \varepsilon_{0 \mathrm{e}}^{2}+\frac{1}{2} \mu \boldsymbol{e}_{\mathrm{e}}: \boldsymbol{e}_{\mathrm{e}}\right] \eta(1-\eta) \\
& \quad-4 A \eta(1-\eta)(0.5-\eta) ; \quad 3 p_{\mathrm{e}}:=\sigma_{\mathrm{e}}: \boldsymbol{I},
\end{aligned}
$$

in which $\sigma_{\mathrm{e}}$ and $\boldsymbol{\sigma}_{\mathrm{st}}$ are the elastic stress and surface tension, $\boldsymbol{\sigma}^{\mathrm{e}}=p_{\mathrm{e}}$ for volumetric transformation strain and $\sigma^{e}=\boldsymbol{k} \cdot \boldsymbol{\sigma}_{\mathrm{e}} \cdot \boldsymbol{k}$ for uniaxial transformation strain. For a phase-equilibrium condition in the stressfree case, $(\beta / 2)|\nabla \eta|^{2}=\breve{\psi}^{\theta}$ (ref. 20), and we have $\boldsymbol{\sigma}_{\text {st }}=\beta|\nabla \eta|^{2}(\boldsymbol{I}-\boldsymbol{k} \otimes \boldsymbol{k})$, that is, it represents two equal normal stresses along the interface. Thus, $\sigma_{\text {st }}$ is consistent with the SIA, unlike previous approaches ${ }^{11-14}$, which resolves a long-standing problem in PFA for melting.

In contrast to previous works on melting and other transformations in solids ${ }^{14,18}$, the gradient operator $\nabla$ with respect to the deformed state was used, and the finite-strain factor $J$ was included in the proper places. Although use of $\nabla$ is natural for liquids ${ }^{11-13}$, this is not the case for solids. One of the unexpected points is that even for small strains, one cannot assume that $J \simeq 1$ and $\nabla \simeq \stackrel{\circ}{\nabla}$, because this leads to zero surface tension. The same thermodynamic procedure $^{19}$ that led to equation (1) also results in the boundary conditions

$$
J \frac{\partial \psi}{\partial \nabla \eta} \cdot \boldsymbol{n}=\beta \nabla \eta \cdot \boldsymbol{n}=-\frac{d \gamma}{d \eta}, \quad \sigma_{n}=\frac{2 \gamma(\eta)}{R}+\bar{p}
$$

in which $\boldsymbol{n}$ is the unit normal to the boundary, $\sigma_{n}$ is the normal to interface stress, $1 / R$ is the mean curvature and $\bar{p}$ is the external pressure. Equation (8) represents a generalization for the 3 -D case and coupling with mechanics of known condition ${ }^{15,16}$. We require the following properties of the surface energy: $\gamma(0)=\gamma_{1} ; \gamma(1)=\gamma_{s}$; $\mathrm{d} \gamma(0) / \mathrm{d} \eta=\mathrm{d} \gamma(1) / \mathrm{d} \eta=0$. The last equation guarantees that homogeneous melt $(\eta=0)$ and solid $(\eta=1)$ satisfy equation (8) and removes contradiction in the known papers ${ }^{15,16}$ (see Fig. 2). These properties are met for polynomial $\gamma(\eta)=\gamma_{1}+\Delta \gamma\left(\mathrm{a} \eta^{2}+(4-2 \mathrm{a}) \eta^{3}+\right.$ $\left.(a-3) \eta^{4}\right)$ of the lowest degree with a parameter $a$. One can demonstrate that a criterion of barrierless surface-induced melt nucleation is consistent with a sharp-interface condition, $\Delta \gamma>\gamma_{\mathrm{s}-1}$, when $a=3$. 
Then $\gamma(\eta)=\gamma_{1}+\Delta \gamma \phi(\eta)$. If surface energy does not change during melting, then $\gamma=$ const and equation (8) reduces to traditional boundary condition $\nabla \eta \cdot \boldsymbol{n}=0$.

We focus on Al nanoparticles; material parameters are given in Supplementary Discussion. In particular, $\theta_{\mathrm{e}}=933.67 \mathrm{~K}, \theta_{\mathrm{c}}=0.8 \theta_{\mathrm{e}}=$ $746.9 \mathrm{~K}$ and $\theta_{\mathrm{i}}=1.2 \theta_{\mathrm{e}}=1120.4 \mathrm{~K}, \delta_{\mathrm{e}}=2.97 \mathrm{~nm}$. Note that all material parameters have been obtained for a macroscopic sample with no fitting parameters left. None of our developments (coherent interface with uniaxial transformation strain, expressions for free energy that result in correct expression for surface tension, and expression for surface energy) requires additional material parameters. First, homogeneous heating of particles of radius $R$ was considered and stationary solutions were found for each temperature. The thickness $h$ of pre-molten and completely molten surface layer (determined by an interphase radius $r_{\mathrm{i}}$, that is, by point with $\eta=0.5$ ) have been plotted versus $\theta_{\mathrm{e}}-\theta$ (Fig. 3). At melting temperature, $\theta_{\mathrm{m}}$, the stationary, two-phase solution ceases to exist and interface propagates to the centre.

Size dependence of melting temperature. In Figure 4, the lowest curve is for GL equation only, that is, for neglected mechanics. Below radius $R^{\star}=6.12 \mathrm{~nm}$, results for volumetric $\boldsymbol{\varepsilon}_{t}$ give slightly lower $\theta_{\mathrm{m}}$

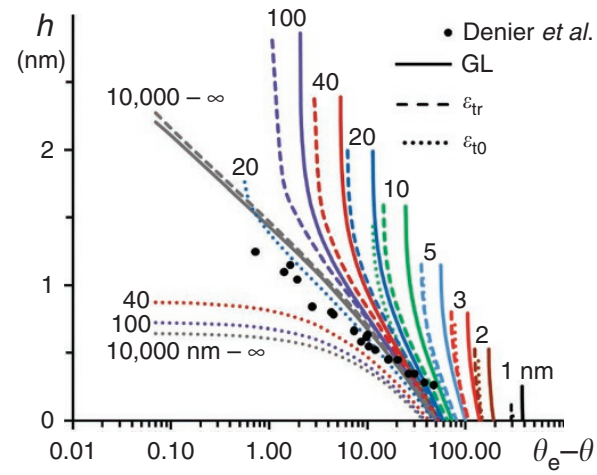

Figure 3 | Thickness of molten surface layer versus temperature for different particle radii. Each colour represents a particle radius shown in nm near the curves. Solid lines are results of the GL model without mechanics. Dotted and dashed lines are obtained for coupled GL and mechanics model with volumetric and uniaxial transformation strains, respectively. Black dots are experimental data' obtained using mediumenergy ion scattering. Experimental points for a plane surface are close to the calculations for infinite radius for $G L$ and coupled models with uniaxial transformation strain. All curves, excluding those interrupted at $0.1 \mathrm{~K}$, are ended at the melting temperature.

a

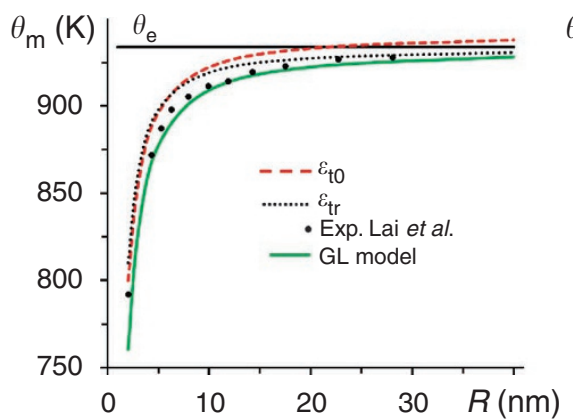

and are closer to the experimental points, whereas for $R>R^{\star}$ results for uniaxial $\boldsymbol{\varepsilon}_{t}$ are much lower. For $R>21.2 \mathrm{~nm}$ and volumetric $\boldsymbol{\varepsilon}_{t}$, $\theta_{\mathrm{m}}>\theta_{\mathrm{e}}$. This is in contrast to the experiments for flat interface, for which $\theta_{\mathrm{m}}=\theta_{\mathrm{e}}$. That is why uniaxial transformation strain was used, in contrast to known models ${ }^{11-13}$. For neglected mechanics and for uniaxial $\boldsymbol{\varepsilon}_{t}$, melting temperature tends to $\theta_{\mathrm{e}}$ for infinite radius. Experimental points are between curves with neglected mechanics and for uniaxial $\boldsymbol{\varepsilon}_{t}$ for $R>R^{\star}$ and for volumetric $\boldsymbol{\varepsilon}_{t}$ for $R<R^{\star}$. Coupling with mechanics for $2-3 \mathrm{~nm}$ particles increases the melting temperature by $30-40 \mathrm{~K}$ (see also Fig. 5 ) and makes it closer to the experiments. Surprisingly, our results are in better correspondence with experiments than known MD approaches (Fig. 4b). Thus, we can conclude that the model of coherent solid-melt interface has good potential for the description of experiments; traditional volumetric $\boldsymbol{\varepsilon}_{t}$ is not adequate for large particles, that is, there is interface restructuring, driven by the internal stress relaxation, and a thermodynamic and kinetic theory for $\boldsymbol{e}_{t}$ should be developed.

Surface premelting and melting. As shown in Figure 3, for a plane interface $(R \rightarrow \infty)$, plots for thickness of the molten layer versus $\theta$ for GL and uniaxial $\boldsymbol{\varepsilon}_{t}$ almost coincide and are very close to experimental points, which justifies the validity of our model for surface energy. Results for volumetric $\boldsymbol{\varepsilon}_{t}$ are inconsistent with experiments. For nanoparticles, $h(\theta)$ plots consist of two parts: an almost straight line with small slope at high temperature with transition to an almost vertical line for temperatures close to $\theta_{\mathrm{m}}$. The maximum thickness of the molten layer is very close for the GL model and the model with uniaxial $\boldsymbol{\varepsilon}_{t}$. Although curves for these models look also close, for some temperatures the difference in thickness is a factor of 2 or larger. For $R \leq 5 \mathrm{~nm}$, curves for volumetric and uniaxial $\boldsymbol{\varepsilon}_{t}$ are very close.

Stationary distributions of interface profiles $\phi(\eta)$ (note that $\phi(\eta)$ rather than $\eta$ describes the variation of all properties, see equation (1)) in particles of $R=1$ and $3 \mathrm{~nm}$ are presented in Figure 5. For all cases, the complete liquid phase $(\eta=0)$ is not reached. For 1 and $3 \mathrm{~nm}$, stationary premelting (that is, $\phi(\eta)<1$ ) occurs in the entire particle rather than at the surface only. Allowing for mechanics increases the melting temperature by $70 \mathrm{~K}$ for the $R=1 \mathrm{~nm}$ particle, mostly due to pressure induced by surface tension. Note that for $R<1.5 \mathrm{~nm}$, one has $\theta_{\mathrm{m}}<\theta_{\mathrm{c}}$, that is, the particle melts even when bulk melt is unstable.

Surface tension. Distributions of radial stresses along $r$ are shown in Figure 6. When internal elastic stresses can be neglected (for equal thermoelastic properties of phases and $\mu=0$, that is, for liquid-liquid transformation, or for $\boldsymbol{\varepsilon}_{t}=0$ ), our calculations at $\theta_{\mathrm{e}}$ reproduce the Laplace relationship for jump in radial stresses $\Delta \sigma_{\mathrm{r}}=-2 \gamma_{\mathrm{s}-1} / r_{i}$ within an error of $0.14 \%$ for $r_{i}>11 \mathrm{~nm}$ and $1.03 \%$ at $r_{i}=5 \mathrm{~nm}$. For actual solid- b

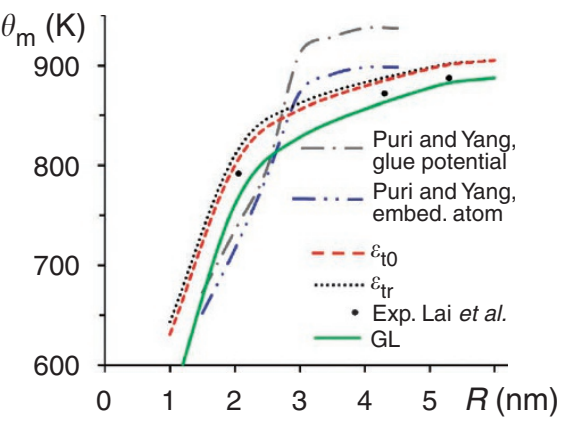

Figure 4 | Melting temperature of Al versus particle radius. (a) Curves are calculated using three models: GL equations only (GL, green line), GL equation coupled to mechanics with volumetric $\left(\varepsilon_{t 0}\right)$ (red line) and uniaxial $\left(\varepsilon_{\mathrm{tr}}\right)$ (black dotted line) transformation strain. Dots are experimental data from Lai et al. ${ }^{3}$ obtained using thin-film differential scanning calorimetry. The horizontal line is the equilibrium temperature (the same curve designations are used in b). (b) Molecular dynamics results are added for particles with radii in the 1-6 nm range. Gray and blue lines are MD results ${ }^{5}$ using glue and embedded atom potentials, respectively. 
a

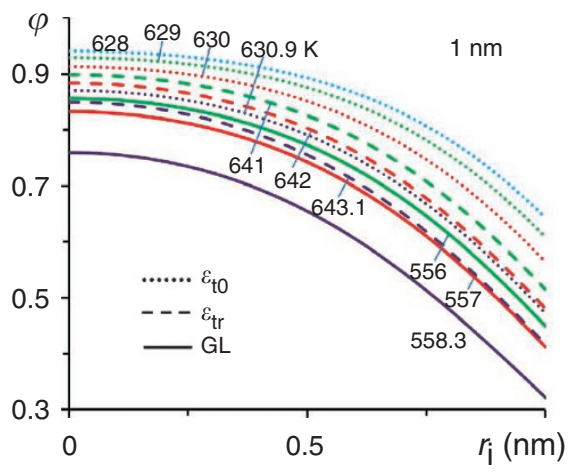

b

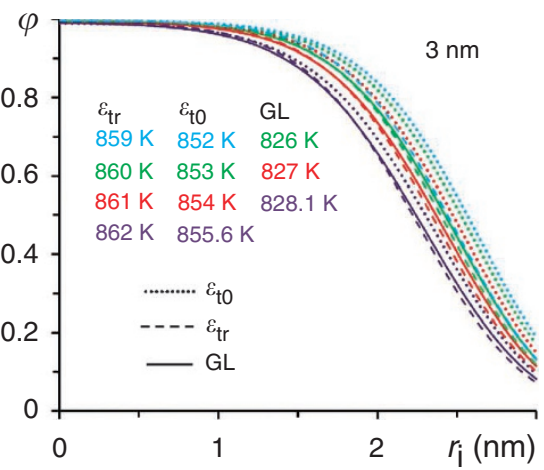

Figure 5 | Stationary interface profiles $\phi(\boldsymbol{\eta})$ for Al particles for different temperatures. (a) $R=1 \mathrm{~nm} ;(\mathbf{b}) R=3 \mathrm{~nm}$. Solid lines are the results obtained with the $\mathrm{GL}$ model. Dotted and dashed lines are obtained using coupled GL and mechanics models with volumetric and uniaxial transformation strains, respectively. Different temperatures are considered for each model, which are designated on the graphs. The lowest curve for each model corresponds to the melting temperature.

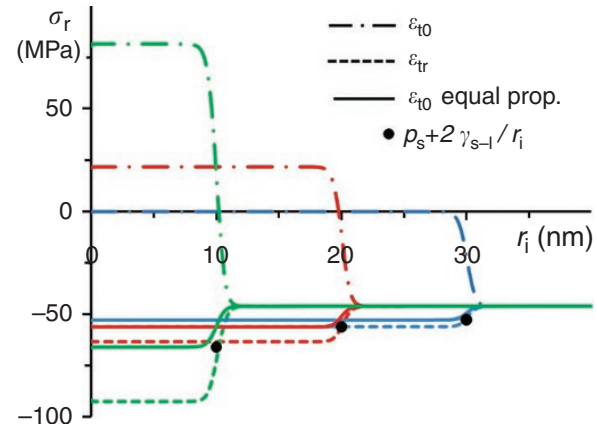

Figure 6 | Distribution of radial stresses for three interface positions. The blue line corresponds to the interface radius at $30 \mathrm{~nm}$, the red at $20 \mathrm{~nm}$, and the green at $10 \mathrm{~nm}$. Distributions are calculated at $\theta_{\mathrm{e}}$ using models with volumetric $\left(\varepsilon_{t 0}\right)$ (dotted line) and uniaxial $\left(\varepsilon_{\mathrm{tr}}\right)$ (dashed line) transformation strains, as well as with equal thermoelastic properties of solid and melt (solid line), that is, for liquid-liquid transformation. Dots designate pressures in melt $p_{s}+2 \gamma_{s-} / r_{i}$ based on the Laplace equation that coincides with results of calculations for equal thermoelastic properties of the solid and melt.

melt properties and volumetric $\boldsymbol{\varepsilon}_{t}, \Delta \sigma_{\mathrm{r}}$ does not follow the Laplace relationship and even possesses the opposite sign, causing tension in the solid core (Fig. 6). For uniaxial $\boldsymbol{\varepsilon}_{t}, \Delta \sigma_{\mathrm{r}}=-k 2 \gamma_{\mathrm{s}-1} / r_{i}$ with $k>1$.

Interface profile and energy. For plane interface, $\theta=\theta_{\mathrm{e}}$, and neglected mechanics, the GL equation allows analytical solution for an equilibrium interface:

$$
\eta_{\mathrm{e}}=\left[1+\exp \left(5.54 x / \delta_{\mathrm{e}}\right)\right]^{-1} ; \quad \delta_{\mathrm{e}}=5.54 \sqrt{\beta /(2 A)} ; \quad \gamma_{s-l}=\sqrt{\beta A / 18},
$$

where the interface width is $\delta=\left|r_{1}-r_{\mathrm{s}}\right|$ and $r_{1}$ and $r_{\mathrm{s}}$ are determined from the conditions $\phi(\eta)=0.01$ and 0.99 , respectively. Surface energy in non-equilibrium state $\gamma_{s-l}^{n}$ is defined as an excess energy with respect to solid (where $0.5<\eta \leq 1$ ) and liquid (where $0 \leq \eta \leq 0.5$ )

$$
\gamma_{s-l}^{n}=\left(\int_{0}^{r_{i}}\left(\rho \psi-\rho_{s} \psi_{s}\right) r^{2} \mathrm{~d} r+\int_{r_{i}}^{R-h}\left(\rho \psi-\rho_{l} \psi_{l}\right) r^{2} \mathrm{~d} r\right) / r_{i}^{2} .
$$

With neglected mechanics, interface width is described by equation (9) within $2.5 \%$ error for $r_{i} \geq 2 \delta_{\mathrm{e}}$ and $\theta \leq 1.25 \theta_{\mathrm{e}}$, that is, even above $\theta_{i}$. For $r_{i}=2 \mathrm{~nm}, \delta=3.99 \mathrm{~nm}=1.34 \delta_{\mathrm{e}}$. Mechanics slightly $(<1 \%)$ increases the width for $\theta=\theta_{\mathrm{e}}$, but this difference grows with increasing temperature; for example, $\delta=3.03$ and $3.06 \mathrm{~nm}$ for $r_{i}=30 \mathrm{~nm}$, $\theta=1.2 \theta_{\mathrm{e}}$ and $1.25 \theta_{\mathrm{e}}$, respectively. Still, the interface profile differs

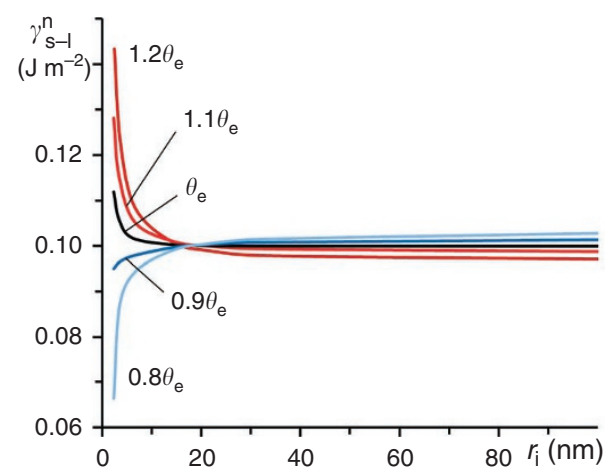

Figure 7 | Non-equilibrium interface energy $\gamma_{s-I}^{n}$ versus interface radius for different temperatures. Results are obtained for both volumetric and uniaxial transformation strains and practically coincide. At the position of $r_{\mathrm{i}}=10 \mathrm{~nm}$, curves from top to bottom correspond to temperatures of $1.2 \theta_{\mathrm{e}^{\prime}} 1.1 \theta_{\mathrm{e}^{\prime}} \theta_{\mathrm{e}^{\prime}} 0.9 \theta_{\mathrm{e}}$ and $0.8 \theta_{\mathrm{e}^{\prime}}$. A crossover in temperature dependence of interface energy for radii below $20 \mathrm{~nm}$ is evident.

from equation (9) within $\Delta \phi=0.03$ error for $r_{i} \geq \delta_{\mathrm{e}} / 2$ and $\theta \leq \theta_{i}$. Interface energy $\gamma_{s-l}^{n}$ versus interface radius for different temperatures is shown in Figure 7. For equilibrium temperature, interface energy starts growing for $r_{i}<10 \mathrm{~nm}$ and maximum deviation from $\gamma_{\mathrm{s}-1}=0.1 \mathrm{Jm}^{-2}$ is $11.2 \%$ for the smallest $r_{i}=2.41 \mathrm{~nm}$, for which full interface exists. For $r_{i}>20 \mathrm{~nm}$ expected decrease in $\gamma_{s-l}^{n}$ with growing temperature is observed. For $r_{i}<20 \mathrm{~nm}$, unexpectedly the opposite relation is found, which means change in sign of the interface entropy. Note that for $r_{i}>4 \delta_{\mathrm{e}} \simeq 12 \mathrm{~nm}$, deviation of $\gamma_{s-l}^{n}$ from $\gamma_{s-1}$ does not exceed $3 \%$ in the entire temperature range $\theta_{c}<\theta<\theta_{i}$, which is well below the inaccuracy of experimental data for interface energy.

Interface velocity. When internal stresses inside an interface are neglected, the following linear relationship is obtained for $r_{i} \gg \delta_{\mathrm{e}}$ between interface velocity $v_{0}$ and the thermodynamic force for interface propagation per unit deformed volume of solid $X$ :

$$
\begin{aligned}
& \left.v_{0}=6 X \chi \sqrt{\beta \rho_{\mathrm{m}} /\left(2 A \rho_{\mathrm{s}}\right.}\right), \\
& X=\frac{\rho_{\mathrm{s}}}{\rho_{0 \mathrm{~s}}} H\left(1-\frac{\theta}{\theta_{\mathrm{e}}}\right)+p_{\mathrm{m}}\left(\frac{\rho_{\mathrm{s}}}{\rho_{\mathrm{m}}}-1\right)-\frac{1}{2}\left(\frac{p_{\mathrm{m}}^{2}}{K_{\mathrm{m}}}-\frac{p_{\mathrm{s}}^{2}}{K_{\mathrm{s}}}\right)+\frac{2 \gamma_{\mathrm{s}-l}}{r_{i}} .
\end{aligned}
$$

It can be seen from Figure 8 that equation (11) describes well (error $\leq 3 \%)$ results of our simulations even for $r_{i}>2 \delta_{\mathrm{e}}$ for neglected internal stresses and for heating rates as high as $10^{13} \mathrm{Ks}^{-1}$. Owing to 


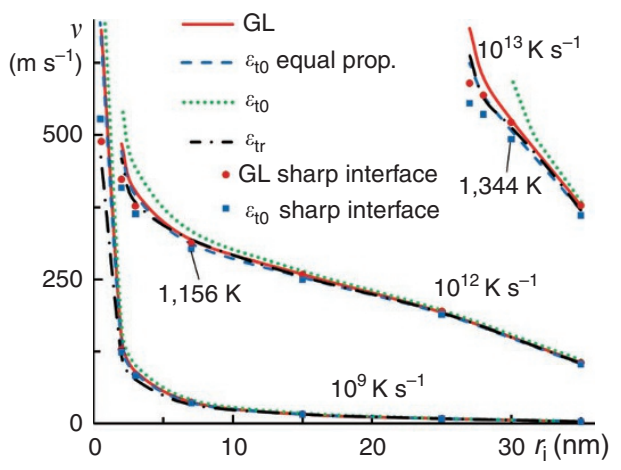

Figure 8 | Interface velocity versus position for three heating rates.

The heating rate is $10^{9} \mathrm{Ks}^{-1}$ at the bottom, $10^{12} \mathrm{Ks}^{-1}$ in the middle, and $10^{13} \mathrm{~K} \mathrm{~s}^{-1}$ at the top. Curves correspond to PFA with different models (red line for GL model, green and black lines for models with volumetric and uniaxial transformation strains, respectively, and blue line for volumetric transformation strain with equal solid and melt properties). Points correspond to SIA, namely, to equation (11).

small particle size, homogeneous temperature is assumed; see the analytical justification in ref. 8. The temperature at some points in Figure 8 significantly exceeds the instability temperature $\theta_{\mathrm{i}}$ and reaches $1344 \mathrm{~K}$ for $10^{13} \mathrm{~K} \mathrm{~s}^{-1}$; that is why interface does not reach the centre of the particle, because homogeneous reduction of $\eta$ in the central part completes melting faster. Even for high compressive and tensile pressures, equation (11) works well when internal stresses are negligible: for external pressure $p=4 \mathrm{GPa}$ and $\theta=1225 \mathrm{~K}$ deviations from simulations for $r_{i}=30$ and $20 \mathrm{~nm}$ are within $2.14 \%$, and for $p=-4 \mathrm{GPa}$ and $\theta=750 \mathrm{~K}$ it is within $1.05 \%$. Velocities for volumetric $\boldsymbol{\varepsilon}_{t}$ are higher, and for uniaxial $\boldsymbol{\varepsilon}_{t}$ they are lower than for the case with $\mu=0$.

\section{Discussion}

Note that the SIA ${ }^{21}$ to melting/solidification at the nanoscale, which includes surface-induced melting and coupled to mechanics, can be applied to our problems down to some radius, which is to be determined by comparison with PFA. However, when the interface region is a significant part of the particle, PFA is more precise and does not require any adjustments. In contrast, SIA should incorporate the size dependence of surface melting (Fig. 3), size and temperature dependence of surface energy (Fig. 7), and surface stresses not equal to surface tension (Fig. 6). To summarize, an advanced phase-field approach to premelting and melting coupled to mechanics is developed. It is applied to study melting deeply in the region of metastability and complete instability of solid and melt, intermediate states at the surface and in few nm-size particles, scale effect, and non-equilibrium thermodynamic and kinetic properties. The importance of mechanics effects (even without external pressure) is elucidated. Crossover in temperature dependence of the interface energy for radii below $20 \mathrm{~nm}$ and violation of the Laplace relationship for the jump in pressure is obtained. Conceptual validity of the coherent solid melt is proven and necessity for its further development, namely, formulation of the evolution equation for the deviatoric part of $\boldsymbol{\varepsilon}_{t}$, is demonstrated.

\section{Methods}

The finite element method code COMSOL Multiphysics was utilized to solve coupled equations (2)-(8). Quadratic Lagrangian elements are used for both the mechanical and GL differential equations. In all, 15-30 elements per interface width and an adaptive time step with a minimum of 1,000 time steps for a typical non-stationary problem are used. To check the model, interface profiles are compared with analytical results for plane interface at equilibrium temperature ${ }^{22}$. Even with an interface radius of $30 \mathrm{~nm}$, difference between planar analytical solution and COMSOL GL result is less than $0.1 \%$. Also, the solid-liquid interface energy in $\mathrm{Al}$ practically coincides with its analytical value of $0.1 \mathrm{~J} \mathrm{~m}^{-2}$, see Figure 7 .

\section{References}

1. Denier van der Gon, A. W., Smith, R. J., Gay, J. M., O'Connor, D. J. \& van der Veen, J. F. Melting of Al surfaces. Surf. Sci. 227, 143-149 (1990).

2. Henson, B. F. \& Robinson, J. M. Dependence of quasiliquid thickness on the liquid activity: a bulk thermodynamic theory of the interface. Phys. Rev. Lett. 92, 246107 (2004).

3. Lai, S. L., Carlsson, J. R. A. \& Allen, L. H. Melting point depression of Al clusters generated during the early stages of film growth: nanocalorimetry measurements. Appl. Phys. Lett. 72, 1098-1100 (1998).

4. Levitas, V. I., Pantoya, M., Chauhan, G. \& Rivero, I. Effect of the alumina shell on the melting temperature depression for nano-aluminum particles. J. Phys. Chem. C 113, 14088-14096 (2009).

5. Puri, P. \& Yang, V. Effect of particle size on melting of aluminum at nano scales. J. Phys. Chem. C 111, 11776-11783 (2007).

6. Ivanov, D. S. \& Zhigilei, L. V. Combined atomistic-continuum modeling of short pulse laser melting and disintegration of metal films. Phys. Rev. B 68, 064114 (2003).

7. Iglev, H., Schmeisser, M., Simeonidis, K., Thaller, A. \& Laubereau, A. Ultrafast superheating and melting of bulk ice. Nature 439, 183-186 (2006).

8. Levitas, V. I., Asay, B. W., Son, S. F. \& Pantoya, M. Mechanochemical mechanism for fast reaction of metastable intermolecular composites based on dispersion of liquid metal. J. Appl. Phys. 101, 083524 (2007).

9. Grinfeld, M. A. Thermodynamic Methods in the Theory of Heterogeneous Systems (Longman, 1991).

10. Delogu, F. Mechanistic aspects of homogeneous and heterogeneous melting processes. J. Phys. Chem. B 110, 12645-12652 (2006).

11. Lowengrub, J. \& Truskinovsky, L. Quasi-incompressible Cahn-Hillard fluids and topological transitions. Proc. R. Soc. London A 454, 2617 2654 (1998).

12. Anderson, D. M., McFadden, G. B. \& Wheeler, A. A. A phase-field model of solidification with convection. Physica D 135, 175-194 (2000).

13. Anderson, D. M., McFadden, G. B. \& Wheeler, A. A. A phase-field model with convection: sharp-interface asymptotics. Physica D 151, 305-331 (2001).

14. Slutsker, J., Thornton, K., Roytburd, A. L., Warren, J. A. \& McFadden, G. B. Phase field modeling of solidification under stress. Phys. Rev. B 74, 014103 (2006).

15. Pluis, B., Frenkel, D. \& Van der Veen, J. F. Surface-induced melting and freezing. 2. A semiempirical Landau-type model. Surf. Sci. 239, 282-300 (1990).

16. Lipowsky, R. Critical surface phenomena at 1st-order bulk transitions. Phys. Rev. Lett. 49, 1575-1578 (1982).

17. Chang, J. \& Johnson, E. Surface and bulk melting of small metal clusters. Philos. Mag. 85, 3617-3627 (2005).

18. Levitas, V. I., Levin, V. A., Zingerman, K. M. \& Freiman, E. I. Displacive phase transition at large strains: phase field theory and simulations. Phys. Rev. Lett. 103, 025702 (2009).

19. Levitas, V. I. \& Javanbakht, M. Surface tension and energy in multivariant martensitic transformations: phase-field theory, simulations, and model of coherent interface. Phys. Rev. Lett. 105, 165701 (2010).

20. Levitas, V. I., Preston, D. L. \& Lee, D. W. Three-dimensional Landau theory for multivariant stress-induced martensitic phase transformations. Phys. Rev. B 68, 134201 (2003)

21. Hoyt, J. J. Effect of stress on melting and freezing in nanopores. Phys. Rev. Lett. 96, 045702 (2006).

22. Levitas, V. I., Lee, D. W. \& Preston, D. L. Interface propagation and microstructure evolution in phase field models. Int. J. Plast. 26, 395-422 (2010).

\section{Acknowledgments}

The support of the National Science Foundation, Office of Naval Research, Air Force Office of Scientific Research, and Iowa State University is gratefully acknowledged.

\section{Author contributions}

V.L. proposed the research and developed theory. K.S. performed the phase field simulations.

\section{Additional information}

Supplementary Information accompanies this paper at http://www.nature.com/ naturecommunications

Competing financial interests: The authors declare no competing financial interests. Reprints and permission information is available online at http://npg.nature.com/ reprintsandpermissions/

How to cite this article: Levitas, V.I. \& Samani. K. Size and mechanics effects in surfaceinduced melting of nanoparticles. Nat. Commun. 2:284 doi: 10.1038/ncomms1275 (2011). 